University of Wollongong

Research Online

Faculty of Engineering and Information

Faculty of Engineering and Information

Sciences - Papers: Part B

Sciences

2020

\title{
Air Void Detection Using Variational Mode Decomposition With Low Rank
}

Fok Hing Chi Tivive

University of Wollongong, tivive@uow.edu.au

Abdesselam Bouzerdoum

University of Wollongong, bouzer@uow.edu.au

Shivakumar Karekal

University of Wollongong, skarekal@uow.edu.au

Follow this and additional works at: https://ro.uow.edu.au/eispapers1

Part of the Engineering Commons, and the Science and Technology Studies Commons

Research Online is the open access institutional repository for the University of Wollongong. For further information contact the UOW Library: research-pubs@uow.edu.au 


\title{
Air Void Detection Using Variational Mode Decomposition With Low Rank
}

\author{
Abstract \\ This paper presents an air-void detection technique for air-coupled radar, which emits electromagnetic \\ waves to interrogate an air-void inside a medium or between two media. The reflections from the air- \\ medium interfaces are usually corrupted by air-coupling, antenna ringing, and internal reflections, \\ rendering air-void detection very difficult or, in certain cases, impossible. The proposed method exploits \\ the low-rank structure of the background clutter to suppress these nuisance signals. A variational mode \\ decomposition model is developed to extract the backscattering at different air-medium interfaces as \\ signal modes. Real experiments are conducted using a stepped frequency radar. The experimental results \\ show that the proposed method can detect air-gap between two sand blocks.

\section{Keywords} \\ rank, void, detection, variational, mode, decomposition, low, air \\ Disciplines \\ Engineering | Science and Technology Studies \\ Publication Details \\ F. Tivive, A. Bouzerdoum \& S. Karekal, "Air Void Detection Using Variational Mode Decomposition With \\ Low Rank," IEEE Sensors Journal, vol. 20, (5) pp. 2600-2607, 2020.
}




\title{
Air Void Detection using Variational Mode Decomposition with Low Rank
}

\author{
Fok Hing Chi Tivive, Member, IEEE, Abdesselam Bouzerdoum, Senior Member, IEEE, and Shivakumar Karekal
}

\begin{abstract}
This paper presents an air-void detection technique for air-coupled radar, which emits electromagnetic waves to interrogate an air-void inside a medium or between two media. The reflections from the air-medium interfaces are usually corrupted by air-coupling, antenna ringing, and internal reflections, rendering air-void detection very difficult or, in certain cases, impossible. The proposed method exploits the low-rank structure of the background clutter to suppress these nuisance signals. A variational mode decomposition model is developed to extract the backscattering at different air-medium interfaces as signal modes. Real experiments are conducted using a stepped frequency radar. The experimental results show that the proposed method can detect air-gap between two sand blocks.
\end{abstract}

Index Terms-stepped-frequency radar, variational mode decomposition, air void detection, low-rank, background clutter removal.

\section{INTRODUCTION}

Ground penetrating radar (GPR) is a non-invasive, nondestructive sensing technology that interrogate solid medium such as ground, rocks or concrete structures to detect the reflection that occurs at an interface, which is caused by a change in the dielectric permittivity of the medium or an object inside the medium. GPR has been used in many applications, including groundwater exploration [1], fracture detection [2], pavement condition assessment [3], railway ballast assessment [4], mine detection [5], just to name a few. In the mining industry, several GPR systems have been developed to provide technological solutions for reducing extraction cost and improving mining safety. In [6], an impulse GPR was used to measure the thickness of coal seam for maintaining a defined coal mining horizon. The coal seam thickness was classified using bispectrum features extracted from the radar signal. Benter et al. determined over-sized fragments, which can block crusher activities, by estimating the bulk density of rock piles using a GPR system [7]. They measured the downshift of the centroid frequency of the radar pulse to determine the density of the rock pile. Thomas and Roy, on the other hand, employed a stepped-frequency radar to estimate

F. H. C. Tivive is with the School of Electrical, Computer and Telecommunications Engineering, University of Wollongong, Northfields Avenue, Wollongong NSW 2522, Australia (Email: tivive@uow.edu.au).

A. Bouzerdoum is with the Information and Computing Technology Division, College of Science and Engineering, Hamad Bin Khalifa University, Doha, Qatar, and the School of Electrical, Computer and Telecommunications Engineering, University of Wollongong, Northfields Avenue, Wollongong NSW 2522, Australia.

S. Karekal is with School of Civil, Mining and Environmental Engineering, University of Wollongong, Northfields Avenue, Wollongong NSW 2522, Australia. thin coal layers, where a high resolution time delay estimation technique is applied for interface detection [8]. Triltzsch et al. used a stepped-frequency radar for detecting separations and clay seams in potash mines [9]. Recently, there has been an increasing demand for using stepped-frequency radars in mining applications to achieve high resolution. Contrary to an impulse radar, a stepped-frequency radar overcomes the power and bandwidth limitations and has a better signal-to-noise ratio. A radar system, be it impulse or stepped-frequency, can be operated into two modes: ground-coupled and air-coupled (also known as, air-launched). In ground-coupled mode, the antennae are placed close the ground surface to achieve better penetration. With an air-coupled GPR, the antenna array is positioned at a standoff distance, allowing fast data acquisition without damaging the antennae and also providing safety for mining personnel. However, the received radar signal is corrupted by the direct coupling, the direct reflection from the surface of the medium, and its internal reflections. These unwanted signals overwhelms the target echoes, rendering the radar signal analysis very challenging.

This paper presents a technique for air-coupled steppedfrequency radar to detect the air-gap between two media while removing the background clutter, which includes air-coupling and internal reflections (i.e., medium reverberations). The background clutter, which has similar characteristics across the antennae, tends to reside in a low-rank subspace. The reflections from the air-medium interfaces can be regarded as echoes with different travel time delays. The proposed method extracts a low-rank representation of the background clutter, using a synthesis dictionary, and employs variational mode decomposition to represent the scattering beneath the medium as signal modes.

The remainder of the paper is organized as follows. Section II presents the proposed radar signal processing method based on a variational model with low-rank constraint. Section III presents experimental results that validate the effectiveness of the proposed method, followed by concluding remarks in Section IV.

\section{Air-Gap Detection Technique}

This section describes the proposed air-gap detection method. First, the signal model for an air-coupled stepped frequency radar is presented in Section II-A. Then, the variational mode decomposition model with low-rank constraint is introduced in Section II-B. 


\section{A. Stepped-Frequency Radar Signal Model}

Consider an air-coupled stepped-frequency radar for sensing. The array aperture consists of $N$ transceivers. Each transceiver emits a wideband stepped frequency signal comprising $M$ frequencies, equally spaced over the sensing bandwidth. Suppose the radar is placed at a standoff distance $Z_{\text {off }}$ from two media, which are placed one behind the other with an air-gap of size $\Delta Z$, creating four air-medium interfaces (i.e., $G=4$ ). The target signal, which comprises the reflections from these interfaces, for the $m$ th frequency at the $n$th transceiver location $y_{n}^{\mathrm{t}}(m)$ can be written as

$$
y_{n}^{\mathrm{t}}(m)=\sum_{i=1}^{G} \sigma_{i} \exp \left(-j 2 \pi f_{m} \tau_{n, i}\right)
$$

where $\sigma_{i}$ is the complex reflectivity of the $i$ th interface, $\tau_{n, i}$ is the two-way propagation delay between the $n$th transceiver and the $i$ th interface, and $G$ is the number of air-medium interfaces. Apart from the target returns, the received signal also contains the reflections from the surface of the interrogated medium, internal reflections, and noise from the background. Let $y_{n}^{\mathrm{c}}(m)$ and $e_{n}(m)$ denote, respectively, the clutter and noise signals for the $m$ th frequency received at the $n$th antenna. The radar signal can be modeled as

$$
y_{n}(m)=y_{n}^{\mathrm{c}}(m)+y_{n}^{\mathrm{t}}(m)+e_{n}(m),
$$

for $n=1, \ldots, N$ and $m=0, \ldots, M-1$. Since the signals received across the antenna array, $\left\{\mathbf{y}_{n}^{\mathrm{c}}\right\}_{n=1}^{N}$, are highly correlated, they tend to reside in a low-rank subspace. Different subspace decomposition methods such as singular value decomposition (SVD) [10]-[14], principal component analysis (PCA) [15]-[17], and independent component analysis (ICA) [18]-[21] have been proposed to remove the background clutter. The principle of a subspace-based approach is to decompose the radar signal into three different components: clutter, target, and noise. In [12], Riaz et al. applied SVD and assumed that the ground bounces reside in a one-dimensional subspace spanned by the first dominant singular vector. This assumption is valid if the ground surface is smooth and the ground is completely homogeneous. Estimating the number of eigen-components spanning the clutter subspace is difficult since the ground and target singular vectors may interleave. This issue is recently overcome by casting the estimation of the clutter low-rank subspace as a rank minimization problem [5], [22].

\section{B. Variational Mode Decomposition Model with Low-Rank}

The variational mode decomposition (VMD) algorithm, which was proposed by Dragomiretskiy et al. [23], is used to detect the reflection from an interface. For a complex-valued signal, the VMD algorithm estimates the mode bandwidth by performing a heterodyne demodulation to shift the frequency of the mode to baseband and estimating the bandwidth of the mode through the $H^{1}$-norm (Dirichlet energy) of the demodulated signal, i.e., the square $L^{2}$-norm of the gradient [24]. Let $y_{n}^{\mathrm{t}}(m)$ be the complex-valued target signal received at the $n$th transceiver, $u_{n, l}$ be the $l$ th mode extracted from the target signal, $\omega_{n, l}$ be its center frequency, and $L$ denote the number of modes. For signal decomposition, the constrained variational problem can be written as

$$
\begin{array}{r}
\min _{\left\{u_{n, l}(m)\right\},\left\{\omega_{n, l}\right\}} \sum_{l=1}^{L}\left\|\partial_{m}\left[u_{n, l}(m) e^{-j \omega_{n, l} m}\right]\right\|_{2}^{2} \\
\text { s.t. } \sum_{l=1}^{L} u_{n, l}(m)=y_{n}^{\mathrm{t}}(m), \forall n
\end{array}
$$

where the square $L^{2}$-norm is understood as $\|\cdot\|_{2}^{2}=\int|\cdot|^{2} d t$, $\partial_{m}$ is the derivative with respect to $m$, and $*$ denotes the convolution operator.

Here, the background clutter, which is assumed to have lowrank property, is estimated by solving a rank minimization problem. Let $\mathbf{Y}$ denote the matrix containing the antenna signals as its columns. Likewise, the background clutter, the target signal, and the noise components are denoted by $\mathbf{Y}^{\mathrm{c}}=\left[y_{n}^{\mathrm{c}}(m)\right]_{M N}, \mathbf{Y}^{\mathrm{t}}=\left[y_{n}^{\mathrm{t}}(m)\right]_{M N}$, and $\mathbf{E}=\left[e_{n}(m)\right]_{M N}$, respectively. Equation (2) can be written in matrix form as

$$
\mathbf{Y}=\mathbf{Y}^{\mathrm{c}}+\mathbf{Y}^{\mathrm{t}}+\mathbf{E} \text {. }
$$

Suppose the low-rank representation $\mathbf{Z}=\left[z_{n}(i)\right]_{Q N}$ for $n=$ $1, \ldots, N, i=1, \ldots, Q$ is obtained from an overcomplete synthesis dictionary $\mathbf{D}$ of size $M \times Q$, consisting of $Q$ atoms, where $Q>>M$. The rank minimization problem can be written as

$$
\min _{\mathbf{Z}}\|\mathbf{Z}\|_{*} \quad \text { s.t. } \quad \mathbf{Y}^{\mathrm{c}}=\mathbf{D Z} .
$$

Combining the low-rank prior (5) with (3), we obtain the following variational model

$$
\begin{array}{r}
\min _{\mathbf{z},\left\{u_{n, l}(m)\right\},\left\{\omega_{n, l}\right\}}\|\mathbf{Z}\|_{*}+\sum_{n=1}^{N} \sum_{l=1}^{L}\left\|\partial_{m}\left[u_{n, l}(m) e^{-j \omega_{n, l} m}\right]\right\|_{2}^{2} \\
\text { s.t. } \quad \sum_{l=1}^{L} u_{n, l}(m)=y_{n}(m)-\sum_{i=1}^{Q} D(m, i) z_{n}(i) \quad \forall n .
\end{array}
$$

Similar to the VMD technique, a quadratic penalty term and Lagrange multipliers are added to convert (6) into the augmented Lagrangian form:

$$
\begin{gathered}
\mathcal{L}\left(\mathbf{Z}, u_{n, l}(m), \omega_{n, l}, b_{n}(m)\right)=\beta\|\mathbf{Z}\|_{*}+ \\
\alpha \sum_{n=1}^{N} \sum_{l=1}^{L}\left\|\partial_{m}\left[u_{n, l}(m) e^{-j \omega_{n, l} m}\right]\right\|_{2}^{2}+ \\
\left\langle b_{n}(m), y_{n}(m)-\sum_{i=1}^{Q} D(m, i) z_{n}(i)-\sum_{l=1}^{L} u_{n, l}(m)\right\rangle+ \\
\sum_{n=1}^{N}\left\|y_{n}(m)-\sum_{i=1}^{Q} D(m, i) z_{n}(i)-\sum_{l=1}^{L} u_{n, l}(m)\right\|_{2}^{2},
\end{gathered}
$$

where $\beta$ is a penalty parameter controlling the amount of clutter to be removed and $b_{n}(m)$ is the Lagrange multiplier associated with the $n$th antenna. Using the alternating direction method of multipliers (ADMM) technique, we decompose the objective function (7) into the following Subproblems:

$$
\begin{aligned}
& \min _{\mathbf{Z}} \beta\|\mathbf{Z}\|_{*}+ \\
& \sum_{n=1}^{N}\left\|y_{n}(m)-\sum_{i=1}^{Q} D(m, i) z_{n}(i)-\sum_{l=1}^{L} u_{n, l}(m)+\frac{b_{n}(m)}{2}\right\|_{2}^{2}
\end{aligned}
$$




$$
\begin{aligned}
& \min _{u_{n, l}(m)} \alpha\left\|\partial_{m}\left[u_{n, l}(m) e^{-j \omega_{n, l} m}\right]\right\|_{2}^{2}+ \\
& \left\|y_{n}(m)-\sum_{i=1}^{Q} D(m, i) z_{n}(i)-\sum_{l=1}^{L} u_{n, l}(m)+\frac{b_{n}(m)}{2}\right\|_{2}^{2}
\end{aligned}
$$

and

$$
\min _{\omega_{n, l}}\left\|\partial_{m}\left[u_{n, l}(m) e^{-j \omega_{n, l} m}\right]\right\|_{2}^{2} \forall n, l .
$$

Subproblem (8) is a least squares problem regularized by a nuclear norm penalty, which can be concisely written as

$$
\min _{\mathbf{Z}} \beta\|\mathbf{Z}\|_{*}+\|\mathbf{G}-\mathbf{D Z}\|_{F}^{2}
$$

where $\mathbf{G}=\left[y_{n}(m)-\sum_{l=1}^{L} u_{n, l}(m)+0.5 b_{n}(m)\right]_{M N}$. This rank minimization problem can be solved using the linearization and proximal technique proposed by Lin et al. [25]. Firstly, the quadratic term in (11) can be linearized as

$$
\begin{aligned}
&\|\mathbf{G}-\mathbf{D Z}\|_{F}^{2} \approx\left\|\mathbf{G}-\mathbf{D Z}^{k}\right\|_{F}^{2}+\left\langle\nabla \mathbf{Z}^{k}, \mathbf{Z}-\mathbf{Z}^{k}\right\rangle \\
&+\frac{1}{\rho}\left\|\mathbf{Z}-\mathbf{Z}^{k}\right\|_{F}^{2},
\end{aligned}
$$

where $0<\rho<1 / \mathcal{S}\left(\mathbf{D}^{T} \mathbf{D}\right)$ is a proximal parameter, $\mathcal{S}\left(\mathbf{D}^{T} \mathbf{D}\right)$ denotes the spectral radius of $\mathbf{D}^{T} \mathbf{D}$, and $\nabla \mathbf{Z}^{k}$ denotes the gradient of $\left\|\mathbf{G}-\mathbf{D} \mathbf{Z}^{k}\right\|_{F}^{2}$ at $\mathbf{Z}^{k}$. By substituting (12) into (11) and with simple mathematical manipulations, we obtain the following approximation to (11):

$$
\min _{\mathbf{Z}} \beta\|\mathbf{Z}\|_{*}+\frac{1}{\rho}\left\|\mathbf{Z}-\left(\mathbf{Z}^{k}-\rho \nabla \mathbf{Z}^{k}\right)\right\|_{F}^{2} .
$$

Next, Subproblem (13) can be efficiently solved using singular value thresholding (SVT), which performs two operations: SVD and soft-thresholding [26]. Let the element-wise softthresholding operator be defined by

$$
\mathcal{T}(a, b)=\operatorname{sgn}(a) \max (|a|-b, 0),
$$

where $\operatorname{sgn}(\cdot)$ denotes the signum function. The minimization of Subproblem (13) can be performed by applying SVT as follows:

$$
\begin{gathered}
\operatorname{SVD}\left(\mathbf{Z}^{k}-\rho \nabla \mathbf{Z}^{k}\right)=\mathbf{U} \boldsymbol{\Sigma} \mathbf{V}^{H} \\
\mathbf{Z}^{k+1}=\mathbf{U} \mathcal{T}(\boldsymbol{\Sigma}, \beta \rho) \mathbf{V}^{H}
\end{gathered}
$$

where ${ }^{H}$ denotes the Hermitian transpose, $\mathbf{U}$ and $\mathbf{V}$ are unitary matrices, and $\boldsymbol{\Sigma}$ is a diagonal matrix of singular values. Subproblems (9) and (10) are solved in the Fourier domain as

$$
\widehat{u}_{n, l}(\omega)^{k+1}=\frac{\widehat{g}_{n}(\omega)^{k+1}-\sum_{i \neq l} \widehat{u}_{n, i}(\omega)+0.5 \widehat{b}_{n}(\omega)}{1+\alpha\left(\omega-\omega_{n, l}\right)^{2}}
$$

and

$$
\omega_{n, l}^{k+1}=\frac{\int_{-\infty}^{\infty} \omega\left|\widehat{u}_{n, l}(\omega)^{k+1}\right|^{2} d \omega}{\int_{-\infty}^{\infty}\left|\widehat{u}_{n, l}(\omega)^{k+1}\right|^{2} d \omega} \quad \forall n, l,
$$

where $\widehat{g}_{n}(\omega)^{k+1}$ is the Fourier transform of $g_{n}(m)^{k+1}=$ $y_{n}(m)-\sum_{i=1}^{Q} D(m, i) z_{n}(i)^{k+1}$. Lastly, the Lagrange multiplier is updated as

$$
\widehat{b}_{n}(\omega)^{k+1}=\widehat{b}_{n}(\omega)^{k}+\mu\left(\widehat{g}_{n}(\omega)^{k+1}-\sum_{l=1}^{L} \widehat{u}_{n, l}(\omega)^{k+1}\right),
$$

where $\mu>0$ is a fixed step size. Subproblems (8) to (10) are solved alternately until a stopping criterion is met. Then, the target signal $\widetilde{y}_{n}^{\mathrm{t}}(m)$ is estimated as

$$
\widetilde{y}_{n}^{\mathrm{t}}(m)=\sum_{l=1}^{L} \widetilde{u}_{n, l}(m),
$$

where $\widetilde{u}_{n, l}(m)$ is the inverse Fourier transform of $\widehat{u}_{n, l}(\omega)$.

\section{EXPERIMENTAL METHODS AND RESUltS}

The proposed air-void detection method is evaluated on real radar signals, which are obtained from a stepped frequency radar at the radar imaging laboratory of the Centre for Signal and Information Processing (CSIP), University of Wollongong, Australia. The radar system comprises a network analyzer to generate a stepped-frequency signal covering 1 to $4 \mathrm{GHz}$ frequency band with a step size of $7.5 \mathrm{MHz}$ and a scanner to synthesize a 25 -element linear array of length $0.3 \mathrm{~m}$, as shown in Fig. 1(a). Two sand bricks are placed at a standoff distance $Z_{\text {off }}$ in front of the horn antenna; they are positioned to have an air-gap $\Delta Z$ between them, as shown in Fig. 1(b). Each sand brick has a length of $0.4 \mathrm{~m}$, width of $0.12 \mathrm{~m}$ and height of $0.3 \mathrm{~m}$. Several sets of radar measurements were collected to investigate the effectiveness of the proposed air-void detection method at different standoff distances and for different airgap sizes. The standoff distance is varied from $0.6 \mathrm{~m}$ to $1.6 \mathrm{~m}$ with a step size of $0.5 \mathrm{~m}$ and the air-gap $\Delta Z$ is increased from $0.1 \mathrm{~m}$ to $0.2 \mathrm{~m}$ with a step size of $0.05 \mathrm{~m}$. For each standoff distance and air-gap size, a set of 25 stepped-frequency radar signals are acquired.

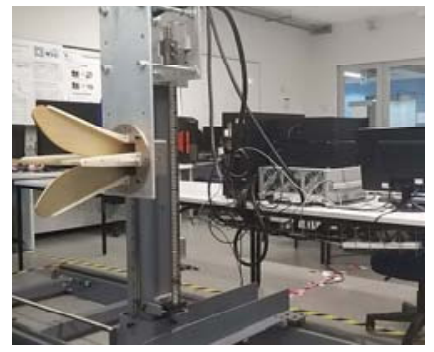

(a)

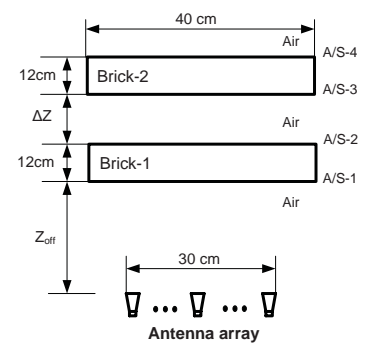

(b)
Fig. 1. The experimental setup of the stepped-frequency radar for air-void detection: (a) image of the stepped-frequency radar system and (b) a schematic layout depicting the positioning of the two sand bricks with air-gap.

The target signals estimated by the proposed variational mode decomposition method are used to form an image, where each horizontal line represents the air-sand (A/S) interface. Here, delay-and-sum (DS) beamforming is used for image formation. Assume the scene is divided into a rectangular grid consisting of $P$ pixels. The magnitude of the $p$ th pixel is computed as

$$
\begin{array}{r}
I(p)=\left|\frac{1}{N M} \sum_{n=1}^{N} \sum_{m=0}^{M-1} \widetilde{y}_{n}^{\mathrm{t}}(m) \exp \left(j 2 \pi f_{m} \tau_{n, p}\right)\right|, \\
\text { for } p=1, \ldots, P,
\end{array}
$$


where $|\cdot|$ denotes the modulus operator and $\tau_{n, p}$ is the focusing delay between the $n$th antenna and the $p$ th pixel.

In addition, the target signal is converted into a high resolution range profile (HRRP), where the peaks depict the locations of the A/S interfaces. Let $\Delta r=c /(2 B)$ denote the radar resolution, where $c$ is the speed of light in free space and $B$ is the bandwidth of the stepped frequency signal. The HRRP $x_{n}(k)$ and the target signal $\widetilde{y}_{n}^{\mathrm{t}}(m)$ are related by the discrete Fourier transform:

$$
x_{n}(k)=\frac{1}{M} \sum_{m=0}^{M-1} \widetilde{y}_{n}^{\mathrm{t}}(m) \exp (j 2 \pi(2 k \Delta r) / c),
$$

for $k=0, \ldots, M-1$ and $n=1, \ldots, N$.

Figure 2 shows the beamformed image and the HRRP plot obtained from the raw radar signals at a standoff distance of $Z_{\text {off }}=1.1 \mathrm{~m}$ and an air-gap size of $\Delta Z=0.1 \mathrm{~m}$. The image in Fig. 2(a) contains strong clutter, which is caused by direct-coupling and antenna ringing. The first two highest peaks of the HRRP in Fig. 2(b) belong to the direct-coupling and antenna ringing. The third peak gives the location of the first $\mathrm{A} / \mathrm{S}$ interface $(\mathrm{A} / \mathrm{S}-1)$. The last two peaks are associated with the second (A/S-2) and fourth $\mathrm{A} / \mathrm{S}$ interfaces (A/S-4). Without background clutter mitigation, it is very difficult to localize the air-gap between Brick-1 and Brick-2, using the image of Fig. 2(a) or the HRRP plot of Fig. 2(b).

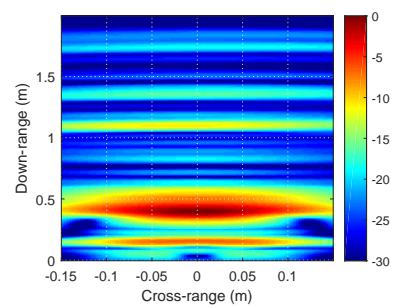

(a)

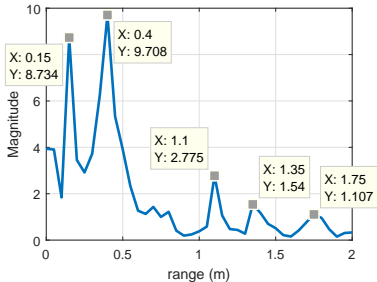

(b)
Fig. 2. Image and HRRP plot obtained directly from the raw radar data: (a) DS-beamformed image, and (b) HRRP of the middle antenna.

Spatial filtering, SVD, PCA, and ICA-based methods are common techniques to remove the background clutter. In spatial filtering, each radar signal is subtracted from a mean vector, which is obtained by averaging all the radar signals. In SVD-based method, the radar data is decomposed into a set of singular vectors and their corresponding singular values. Then, the first few singular vectors associated with the dominant singular values are removed, whereas the remaining singular vectors are used to reconstruct the radar data. Similarly, in PCA-based method, the first few dominant eigen-vectors, which contain the background clutter, are discarded. In the ICA-based method [18], SVD was used to pre-whiten the radar data matrix. Then, the complex-valued joint approximate diagonalization of eigen-matrices (JADE) [27] algorithm was applied to determine the mixing matrix and the independent components. The normalized kurtosis was employed to identify the independent components associated with the target.

Figure 3 depicts the HRRP plots after applying all four existing background clutter removal methods. All the techniques successfully remove the air-coupling and antenna ringing. With spatial filtering, the first two peaks in Fig. 3(a) give, respectively, the locations of the A/S-1 and A/S-2 interfaces. The third peak is related to the A/S-4 interface. Figures 3(b) and (c) depict, respectively, the HRRP plots obtained from SVD and PCA after the removal of the first two dominant components. It is clear that SVD and PCA reveal more peaks, and some of the peaks are closer to the A/S interface locations. Among the four existing clutter removal methods, ICA produce the best HRRP, where the main peaks reveal the location of the A/S-1 interface and the air-gap.

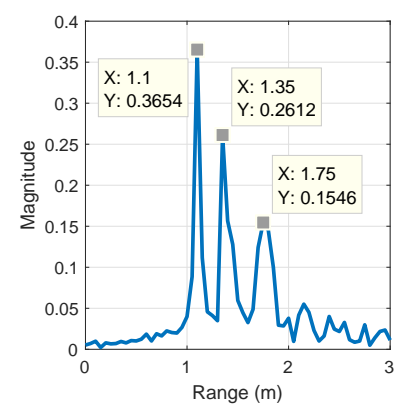

(a)

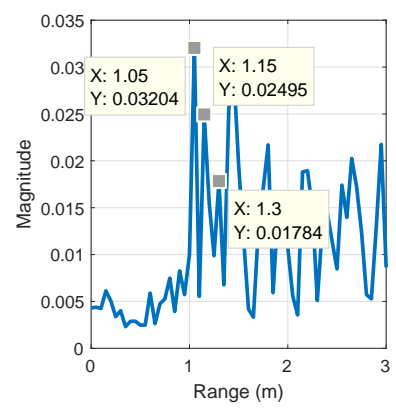

(c)

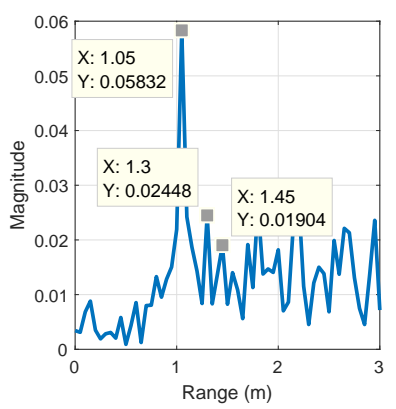

(b)

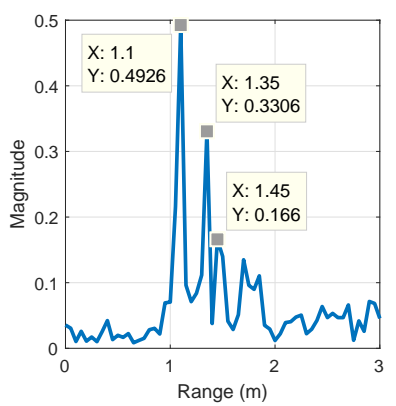

(d)
Fig. 3. HRRP plot after applying (a) spatial filtering, (b) SVD, (c) PCA, and (d) ICA.

Next, the proposed method is applied to detect the air-gap between Brick-1 and Brick-2. The number of modes $L$ and their initial center frequencies $\left\{\omega_{n, l}^{0}\right\}_{l=1}^{L}$ affect the detection of the A/S interfaces. Setting $L$ to a small value can miss some of the $\mathrm{A} / \mathrm{S}$ interfaces, whereas setting $L$ to a large value can generate a lot of false alarms. Therefore, a two-step regularization parameter estimation procedure is employed to find the parameters $L$ and $\left\{\omega_{1}^{0}, \ldots, \omega_{L}^{0}\right\}$. The same initial center frequencies are used for all radar signals. In the first step, the parameter $L$ is set to 100 and the center frequency of each mode is initialized randomly in the range [0.075, 0.125], which corresponds to the range interval of $\left[Z_{\mathrm{off}}, Z_{\mathrm{off}}+1\right]$. Here, the distance $Z_{\text {off }}$ is determined as the range of the first peak in the HRRP plot after applying spatial filtering to remove the antenna ringing. Moreover, the range-of-interest is set to $1 \mathrm{~m}$. After applying a cross-validation procedure, the other parameters are set to: $\beta=0.5\left\|\mathbf{D}^{\dagger} \mathbf{Y}\right\|_{\infty}, \alpha=10^{8}$ and 
$\mu=10^{-5}$. The dictionary $\mathbf{D}$ comprises wavelet packet basis functions obtained from Daubechies wavelets with two level of decompositions. Each atom in the dictionary is normalized to have unit norm. To alleviate the boundary issue in the variational model, a simple mirror extension is performed on the radar signal by adding half of its length on each side. Two stopping criteria are defined: (i) the number of iterations is equal 30 and (ii) the average relative difference between two consecutive extracted modes is below a predefined threshold $\delta$, i.e.,

$$
\frac{\sum_{n=1}^{N} \sum_{l=1}^{L}\left\|\mathbf{u}_{n, l}^{k+1}-\mathbf{u}_{n, l}^{k}\right\|_{2}^{2}}{\sum_{n=1}^{N} \sum_{l=1}^{L}\left\|\mathbf{u}_{n, l}^{k}\right\|_{2}^{2}} \leq \delta,
$$

where $\delta=10^{-5}$ and $\mathbf{u}_{n, l}^{k}=\left[u_{n, l}^{k}(0), \ldots, u_{n, l}^{k}(M-1)\right]^{T}$. The proposed method is applied to estimate the target signals $\left\{\widetilde{y}_{n}^{\mathrm{t}}(m)\right\}_{n=1}^{N}$. Then, an average HRRP is computed as

$$
\widetilde{x}(k)=\frac{1}{M N} \sum_{n=1}^{N}\left|\sum_{m=0}^{M-1} \widetilde{y}_{n}^{\mathrm{t}}(m) \exp (j 2 \pi(2 k \Delta r) / c)\right| .
$$

The average HRRP is re-scaled to the range $[0,1]$ by dividing by the maximum value. A peak detection method is applied to locate peaks with magnitude greater than a pre-defined threshold $\eta$ (here, $\eta=0.2$ ). Let $g_{i}$ denote the range of the $i$ th peak. Suppose there are $J$ detected peaks. Each radar signal is decomposed into $J$ modes, where the initial center frequency of the $i$ th mode $\omega_{i}^{0}$ is determined as

$$
\omega_{i}^{0}=2 \pi \Delta f \frac{2 g_{i}}{c} \quad \text { for } \quad i=1, \ldots, J,
$$

where $\Delta f$ is the step size.

Based on the $J$ modes and their estimated center frequencies, the proposed variational method is applied again to accurately estimate the modes, which are subsequently combined to produce the target signal. Figures 4 to 6 depict the DS images and HRRP plots of the target signals obtained from the proposed method for different standoff distances and air-gap sizes. The HRRP plots clearly show that the proposed method can accurately detect the air-gap between Brick-1 and Brick-2. At a standoff distance of $0.6 \mathrm{~m}$, the proposed method detects all four A/S interfaces, as shown in Fig. 4(b). As the standoff distance increases, more peaks are detected, see Figs. 5(b) and 6(b). These false alarms could be due to the internal reflections or the secondary reflections of the $\mathrm{A} / \mathrm{S}$ interfaces. For comparison, the ICA-based method is also evaluated on the same dataset. Figure 7 shows the HRRP plots obtained from the ICA-based method for different standoff distances and air-gap sizes. Each row of Fig. 7 presents the HRRP plots for different air-gap sizes at a particular standoff distance. The HRRP plots in Figs. 7(a) to (c) show that the ICA-based method has difficulty to detect the air-gap when the antenna is placed close to Brick-1. This is because the directcoupling residuals overwhelm the reflections from the A/S interfaces. Increasing the standoff distance to $1 \mathrm{~m}$ improves the detection of the A/S interfaces. By comparing the HRRP plots in Fig. 7 with those in Figs. 4 to 6, we show that the proposed air-gap detection method achieves better performance than the ICA-based method.

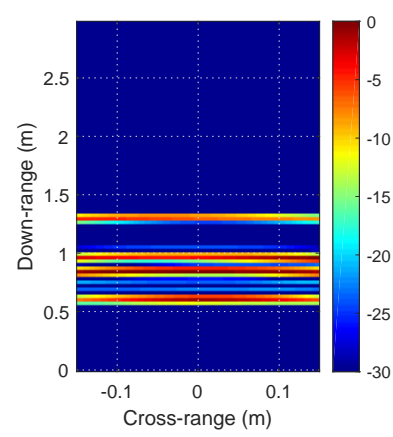

(a)

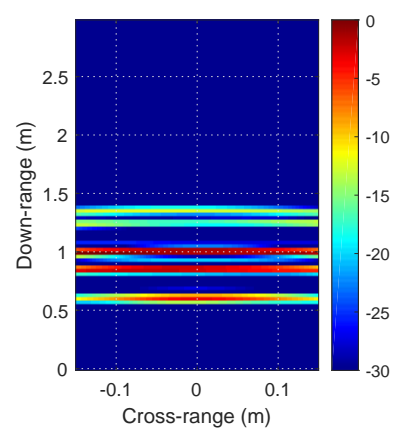

(c)

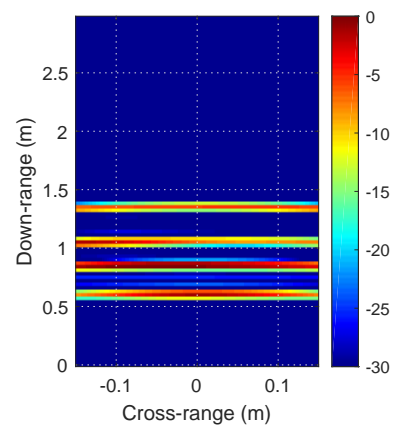

(e)

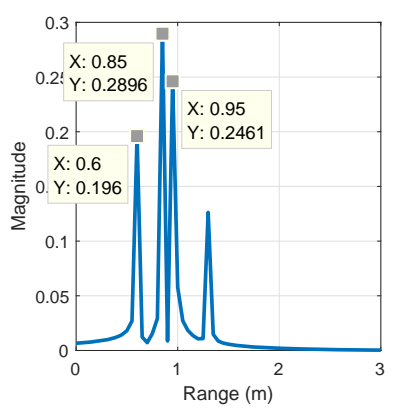

(b)

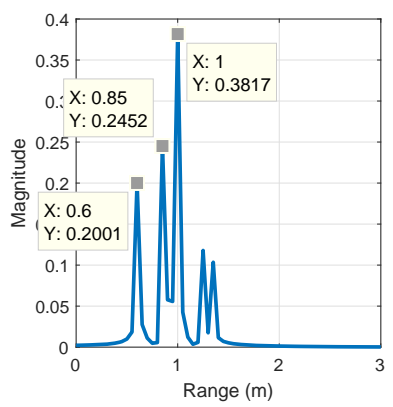

(d)

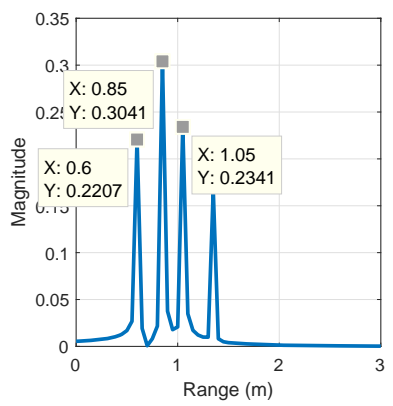

(f)
Fig. 4. Image and HRRP plot obtained from the proposed method at $Z_{\text {off }}=$ $0.5 \mathrm{~m}$ with an air-gap size of (a)-(b) $\Delta Z=0.10 \mathrm{~m}$, (c)-(d) $\Delta Z=0.15 \mathrm{~m}$, and (e)-(f) $\Delta Z=0.20 \mathrm{~m}$.

\section{CONCLUSION}

There is an increase demand of using radar technology in mining industry for estimating coal seam thickness, detecting air-void, and determining over-sized rock fragment. An aircoupled stepped-frequency radar has the advantage of providing high resolution and a stand-off distance when interrogating a region-of-interest or a target. However, the air-coupling, antenna ringing, and the medium reverberations obscure the desired radar returns, rendering the analysis of radar signal very difficult. In this paper, an air-gap detection method was proposed. The proposed method employs a low-rank regularizer to capture the background clutter as a low-rank 


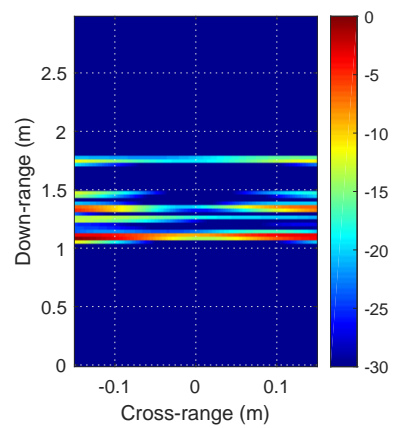

(a)

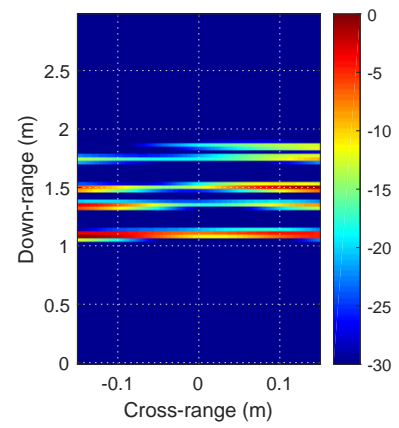

(c)

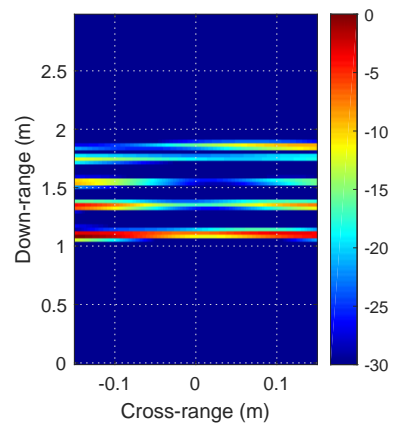

(e)

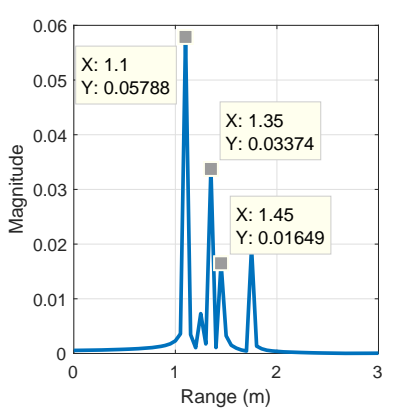

(b)

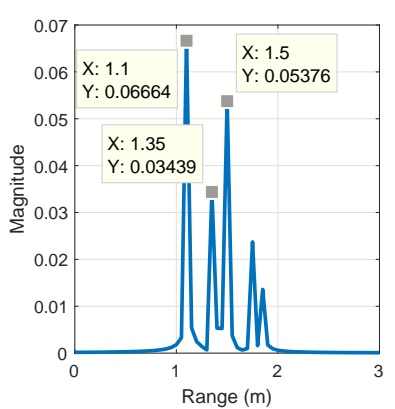

(d)

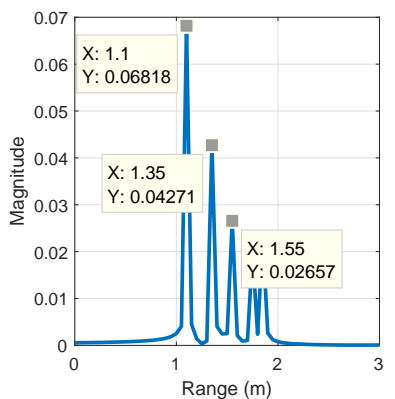

(f)

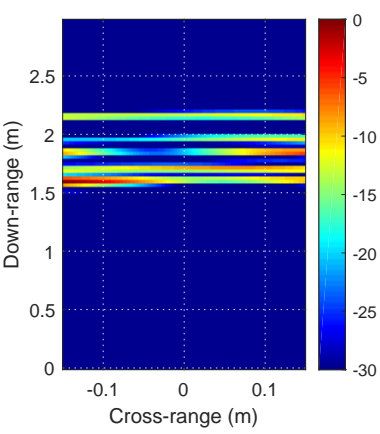

(a)

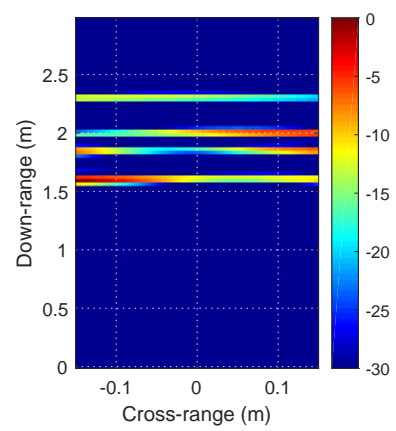

(c)

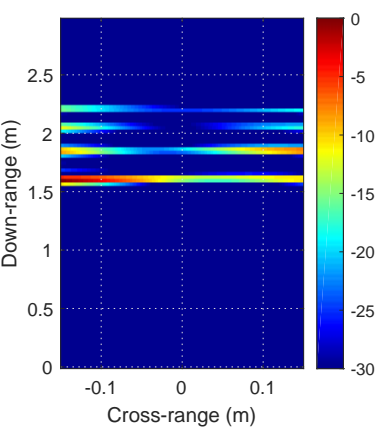

(e)

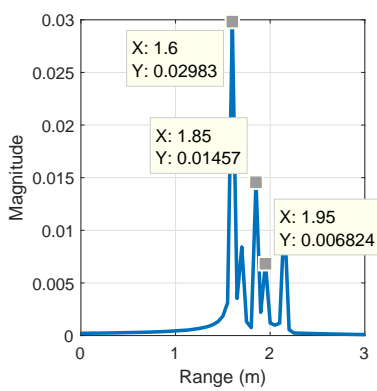

(b)

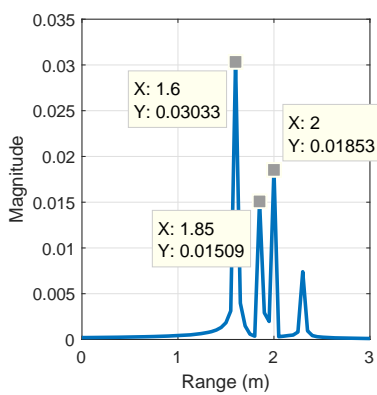

(d)

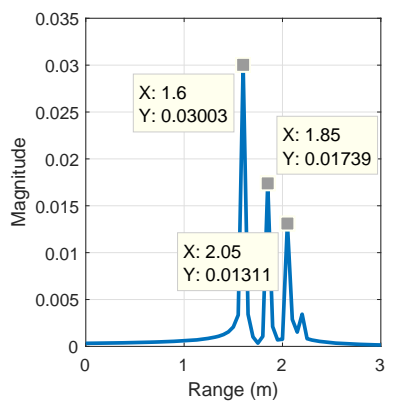

(f)
Fig. 5. Image and HRRP plot obtained from the proposed method at $Z_{\text {off }}=$ $1.0 \mathrm{~m}$ with an air-gap size of (a)-(b) $\Delta Z=0.10 \mathrm{~m}$, (c)-(d) $\Delta Z=0.15 \mathrm{~m}$, and (e)-(f) $\Delta Z=0.20 \mathrm{~m}$.

representation and a variational mode decomposition model to detect the air-medium interfaces as signal modes. Experiments using real stepped frequency signals from 1 to $4 \mathrm{GHz}$ were performed to evaluate the proposed method. Results showed that the air-gap detection method can effectively localize the air-gap of different sizes between two sand bricks at different standoff distances.

\section{ACKNOWLEDGMENT}

This work was supported by a grant from the Australian Research Council (ARC).
Fig. 6. Image and HRRP plot obtained from the proposed method at $Z_{\text {off }}=$ $1.5 \mathrm{~m}$ with an air-gap size of (a)-(b) $\Delta Z=0.10 \mathrm{~m}$, (c)-(d) $\Delta Z=0.15 \mathrm{~m}$ and (e)-(f) $\Delta Z=0.20 \mathrm{~m}$

\section{REFERENCES}

[1] K. Maheswari, P. S. Kumar, D. Mysaiah, K. Ratnamala, M. S. H Rao, and T. Seshunarayana, "Ground penetrating radar for groundwater exploration in granitic terrains: A case study from hyderabad," Journal of the Geological Society of India, vol. 81, no. 6, pp. 781-790, 2013.

[2] M. Lualdi and L. Zanzi, "2D and 3D GPR imaging to map the fractures and to evaluate the integrity of limestone ornamental rocks,' in Symposium on the Application of Geophysics to Eng ineering and Environmental Problems, Nashville, Tenessee, USA, 25-29 Mar. 2003, pp. 613-622.

[3] S. R. Pennock and C. H. J. Jenks, "Road surface and pavement condition assessment by high frequency gpr diffraction," in Proc. of the 15th International Conference on Ground Penetrating Radar, Brussels, Belgium, 30 Jun. - 4 Jul. 2014, pp. 881-886.

[4] W. Shao, A. Bouzerdoum, S. L. Phung, L. Su, B. Indraratna, and 


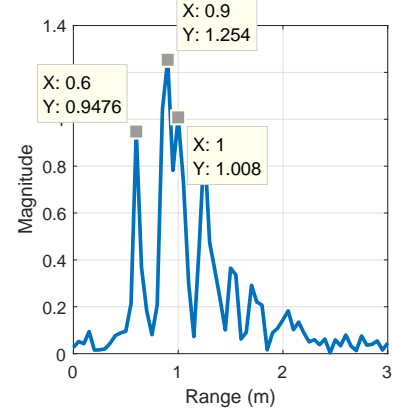

(a)

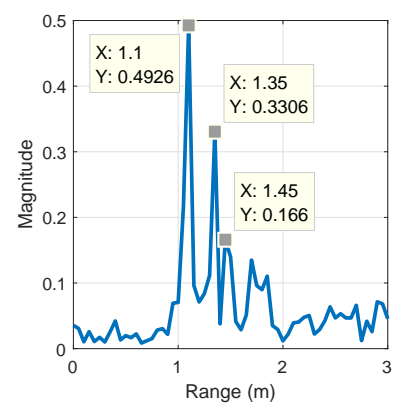

(d)

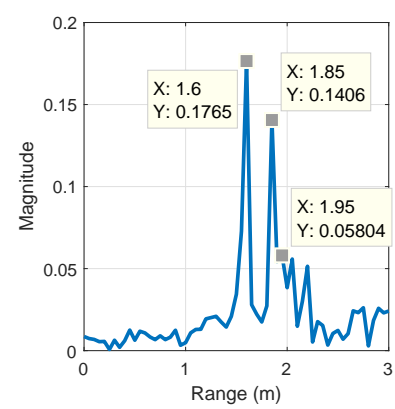

(g)

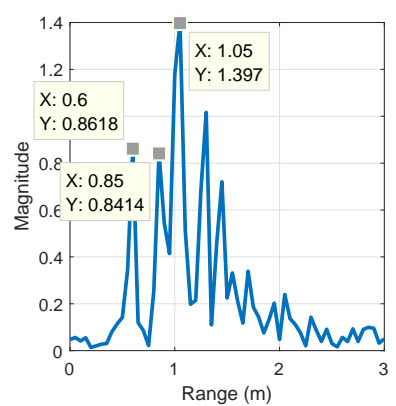

(b)

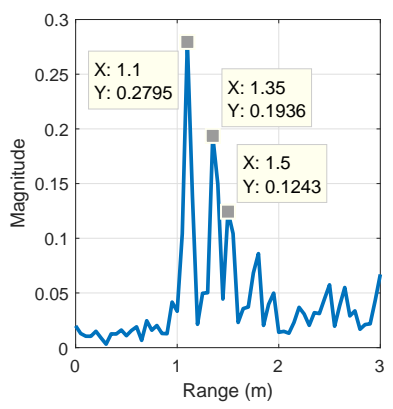

(e)

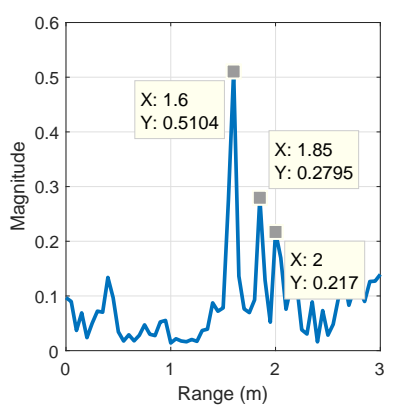

(h)

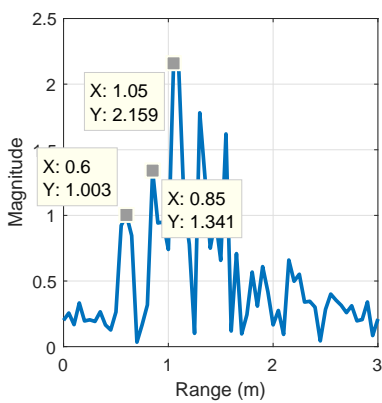

(c)

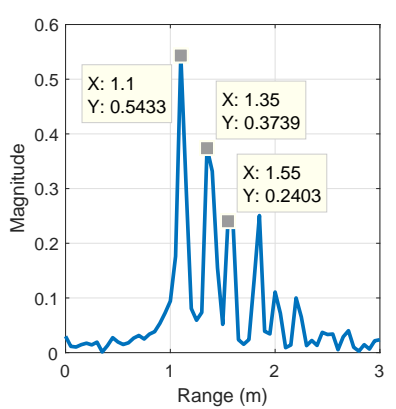

(f)

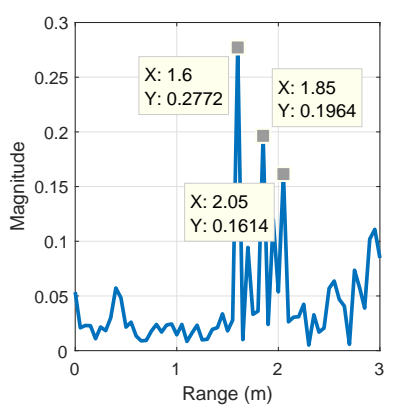

(i)

Fig. 7. HRRP plots obtained from the ICA-based method for different standoff distances and air-gap sizes: (a) $Z_{\mathrm{off}}=0.5 \mathrm{~m}$ and $\Delta Z=0.1 \mathrm{~m}$, (b) $Z_{\text {off }}=0.5 \mathrm{~m}$ and $\Delta Z=0.15 \mathrm{~m}$, (c) $Z_{\text {off }}=0.5 \mathrm{~m}$ and $\Delta Z=0.2 \mathrm{~m}$, (d) $Z_{\text {off }}=1.0 \mathrm{~m}$ and $\Delta Z=0.10 \mathrm{~m}$, (e) $Z_{\text {off }}=1.0 \mathrm{~m}$ and $\Delta Z=0.15 \mathrm{~m}$, (f) $Z Z_{\text {off }}=1.0 \mathrm{~m}$ and $\Delta Z=0.2 \mathrm{~m}$, (g) $Z_{\mathrm{off}}=1.5 \mathrm{~m}$ and $\Delta Z=0.10 \mathrm{~m}$, (h) $Z_{\mathrm{off}}=1.5 \mathrm{~m}$ and $\Delta Z=0.15 \mathrm{~m}$, and (i) $Z_{\mathrm{off}}=1.5 \mathrm{~m}$ and $\Delta Z=0.2 \mathrm{~m}$.

C. Rujikiatkamjorn, "Automatic classification of ground-penetratingradar signals for railway-ballast assessment," IEEE Transactions on Geoscience and Remote Sensing, vol. 49, no. 10, pp. 3961-3972, 2011.

[5] F. H. C. Tivive, A. Bouzerdoum, and C. Abeynayake, "GPR target detection by joint sparse and low-rank matrix decomposition," IEEE Transactions on Geoscience and Remote Sensing, vol. 57, no. 5, pp. 2583-2595, 2018.

[6] A. D. Strange, J. C. Ralston, and V. Chandran, "Application of ground penetrating radar technology for near-surface interface determination in coal mining," in IEEE International Conference on Acoustics, Speech, and Signal Processing, Philadelphia, PA, USA, 23-23 Mar. 2005, pp. 701-704.

[7] A. Benter, M. Antolovich, and W. Moore, "Determining bulk density of mine rock piles using ground penetrating radar frequency downshift," in IEEE International Workshop on Advanced Ground Penetrating Radar, Aachen, Germany, 22-24 Jun. 2011.

[8] S. B. Thomas and L. P. Roy, "Signal processing for coal layer thickness estimation using high-resolution time delay estimation methods," IET
Science, Measurement and Technology, vol. 11, no. 8, pp. 1022-1031, 2017.

[9] G. Triltzsch, H. M. Braun, Y. Krellmann, W. G. Maybee, S. Maloney, P. K. Kaiser, and A. Prugger, "Stepped frequency gpr field trials in potash mines," in Proc. of the Tenth International Conference on Grounds Penetrating Radar, Delft, Netherlands, 21-24 Jun. 2004, pp. 755-758.

[10] B. Cagnoli and T. J. Ulrych, "Singular value decomposition and wavy reflections in ground-penetrating radar images of base surge deposits," Journal of Applied Geophysics, vol. 48, no. 3, pp. 175-182, 2001.

[11] F. Abujarad, A. Jostingmeier, and A. S. Omar, "Clutter removal for landmine using different signal processing techniques," in Proc. Tenth International Conference on Ground Penetrating Radar, Delft, Netherlands, 21-24 Jun. 2004, pp. 697-700.

[12] M. M. Riaz and A. Ghafoor, "Information theoretic criterion based clutter reduction for ground penetrating radar," Progress In Electromagnetics Research B, vol. 45, pp. 147-164, 2012.

[13] M. E. Yavuz, A. E. Fouda, and F. L. Teixeira, "GPR signal enhancement using sliding-window space-frequency matrices," Progress In Electro- 
magnetics Research, vol. 145, pp. 1-10, 2014.

[14] F. H. C. Tivive, A. Bouzerdoum, and M. G. Amin, "A subspace projection approach for wall clutter mitigation in through-the-wall radar imaging," IEEE Transactions on Geoscience and Remote Sensing, vol. 53, no. 4, pp. 2108-2122, 2015.

[15] B. Karlsen, J. Larsen, H. B. D. Sorensen, and K. B. Jakobsen, "Comparison of PCA and ICA based clutter reduction in gpr systems for anti-personal landmine detection," in Proc. 11th IEEE Signal Processing Workshop on Statistical Signal Processing, Singapore, 6-8 Aug. 2001, pp. 146-149.

[16] J. Liu, R. Wu, T. Li, and B. Zhang, "Novel ground bounce removal algorithms based on non-homogeneous detector," in International Conference on Radar, Shanghai, China, 16-19 Oct 2006, pp. 1-5.

[17] Q. Q. Lu, J. X. Pu, X. H. Wang, and Z. H. Liu, "A clutter suppression algorithm for GPR data based on PCA combining with gradient magnitude," Applied Mechanics and Materials, vol. 644-650, pp. 1662-1667, 2014.

[18] B. Karlsen, H. B. D. Sorensen, J. Larsen, and K. B. Jakobsen, "GPR detection of buried symmetrically shaped minelike objects using selective independent component analysis," in Proc. SPIE, Detection and Remediation Technologies for Mines and Minelike Targets VIII, vol. 5089, Orlando, FL, USA, 21-25 Apr. 2003, pp. 375-386.

[19] A. Zhao, Y. Jiang, and W. Wang, "Exploring independent component analysis for GPR signal processing," in Progress In Electromagnetics Research Symposium, Hangzhou, China, 22.26 Aug. 2005, pp. 750-753.

[20] Q. Gao, T. Li, and R. Wu, "A novel KICA method for ground bounce removal with GPR," in International Conference on Radar, Shanghai, China, 16-19 Oct. 2006, pp. 1-4.

[21] W. Chen, W. Wang, J. Gao, J. Xu, and W. Wang, "GPR clutter noise separation by statistical independency promotion," in Proc. 14th Inter national Conference on Ground Penetrating Radar (GPR), Shanghai, China, 4-8 Jun. 2012, pp. 367-370.

[22] M. P. Masarik, J. Burns, B. T. Thelen, J. Kelly, and T. C. Havens, "GPR anomaly detection with robust principal component analysis," in Proc SPIE, Detection and Sensing of Mines, Explosive Objects, and Obscured Targets XX, vol. 9454, Baltimore, Maryland, USA, 20-23 Apr. 2015, pp. $9454141-94541411$

[23] K. Dragomiretskiy and D. Zosso, "Variational mode decomposition," IEEE Transactions on Signal Procesing, vol. 62, no. 3, pp. 531-544, 2014.

[24] S. Yu and J. Ma, "Complex variational mode decomposition for sloppreserving denoising," IEEE Trans. Geoscience and Remote Sensing, vol. 56, no. 1, pp. 586-597, 2018.

[25] Z. Lin, R. Liu, and Z. Su, "Linearized alternating direction method with adaptive penalty for low-rank representation," in Advances in neural information processing systems 24, Granada, Spain, 12-17 Dec. 2011, pp. 612-620.

[26] J.-F. Cai, E. J. Candes, and Z. Shen, "A singular value thresholding algorithm for matrix completion," SIAM Journal on Optimization, vol. 20, no. 4, pp. 1956-1982, 2010.

[27] J. F. Cardoso and A. Souloumiac, "Blind beamforming for non-Gaussian signals," IEE Proceedings F (Radar and Signal Processing), vol. 140, no. 6, pp. 362-370, 1993

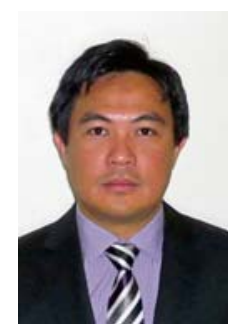

Fok Hing Chi Tivive (M'06) received the BE(Hons) in Telecommunications from Edith Cowan University and the Ph.D. degree in computer engineering from the University of Wollongong, Australia, in 2001 and 2006, respectively. Since 2006, he has been with the School of Electrical Computer and Telecommunications Engineering, University of Wollongong, as a Postdoctoral Research Fellow. His research interests include machine learning, pattern recognition, image processing, ground penetrating radar, and through-the-wall radar imaging.

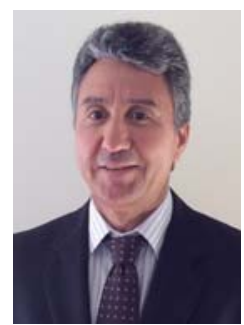

Abdesselam Bouzerdoum (M'89-SM'03 IEEE) graduated with MSEE and Ph.D. degrees, both in Electrical Engineering, from the University of Washington, Seattle, USA. In 1991, he joined Adelaide University, South Australian. In 1998, he moved to Perth, Western Australia, as an Associate Professor with Edith Cowan University. In September 2004 he became Professor of Computer Engineering and Head of School of Electrical, Computer \& Telecommunications Engineering at the University of Wollongong. From 2007 to 2013, he served as Associate Dean (Research) with the Faculty of Informatics, University of Wollongong. He is currently serving as the Head of Information and Computing Technology Division, College of Science and Engineering, Hamad Bin Khalifa University, Qatar, and a Senior Professor of Computer Engineering at the University of Wollongong. Dr. Bouzerdoum held several Visiting Professor Appointments at Institut Galilée, Université Paris-13, LAAS/CNRS, Toulouse, Institut femto-st, Besançon, France, Villanova University, USA, and the Hong Kong University of Science and Technology. From 2009 to 2011, he was a member of the ARC College of Experts and Deputy Chair of the EMI (Engineering, Mathematics and Informatics) panel (2010-2011).

Dr. Bouzerdoum is the recipient of the Eureka Prize for Outstanding Science in Support of Defence or National Security (2011), the Chester Sall Award of IEEE Trans. Consumer Electronics (2005), and a Distinguished Researcher Award (Chercheur de Haut Niveau) from the French Ministry (2001). He served as Associate Editor for 5 International journals, including IEEE TRANS. IMAGE PROCESSING and IEEE TRANS. SYSTEMS, MAN, AND CYBERNETICS (1999-2006). He has published over 360 technical articles and graduated $50 \mathrm{Ph} . \mathrm{D}$. and Research Masters students, and supervised over 60 Honours theses. His research interests include radar imaging and signal processing, image processing, vision, machine learning, and pattern recognition.

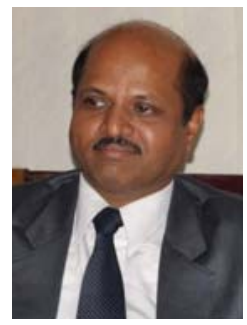

Shivakumar Karekal received the Ph.D. degree in Mining Engineering from the University of Queensland in 2003. He has over 26 years of experience and worked at the University of Queensland, CSIRO, CRC-Mining on permanent positions. He has executed several national and international projects (ACARP, Department of Industry Innovation and Science Research (DIISR/DIISRTE) and AMIRA, Department of Energy on nuclear waste repository design, etc. over several million dollars, and has patents in advanced cutting technology and has published several international journal and conference papers and many technical reports. Many of his research works were confidential in nature. He specializes in (i) advanced computational modelling for both static and dynamics geotechnical engineering problems, (ii) advanced state of art experimental techniques including acoustic emissions, NDT, radar, seismic velocity tomography, fibre optics for progressive fracture and failure. He recently reviewed $\mathrm{PhD}$ thesis related to the development of innovative and very sensitive fibre optics sensor for both static and dynamic applications. He has long standing association with National Institute of Advanced Industrial Science and Technology (AIST), Japan. 